Western University

Scholarship@Western

$3-2013$

Large area periodic, systematically changing, multishape nanostructures by laser interference lithography and cell response to these topographies

Erden Ertorer

Western University

Fartash Vasefi

Western University

Joel Keshwah

Western University

Mohamadreza Najiminaini

Western University

Christopher Halfpap

RheinMain University of Applied Sciences

See next page for additional authors

Follow this and additional works at: https://ir.lib.uwo.ca/anatomypub

Part of the Anatomy Commons, and the Cell and Developmental Biology Commons

Citation of this paper:

Ertorer, Erden; Vasefi, Fartash; Keshwah, Joel; Najiminaini, Mohamadreza; Halfpap, Christopher; Langbein, Uwe; Carson, Jeffrey J. L.; Hamilton, Douglas W.; and Mittler, Silvia, "Large area periodic, systematically changing, multishape nanostructures by laser interference lithography and cell response to these topographies" (2013). Anatomy and Cell Biology Publications. 91.

https://ir.lib.uwo.ca/anatomypub/91 
Authors

Erden Ertorer, Fartash Vasefi, Joel Keshwah, Mohamadreza Najiminaini, Christopher Halfpap, Uwe Langbein, Jeffrey J. L. Carson, Douglas W. Hamilton, and Silvia Mittler 


\section{Biomedical Optics}

\section{Large area periodic, systematically changing, multishape nanostructures by laser interference lithography and cell response to these topographies}

Erden Ertorer

Fartash Vasefi

Joel Keshwah

Mohamadreza Najiminaini

Christopher Halfpap

Uwe Langbein

Jeffrey J. L. Carson

Douglas W. Hamilton

Silvia Mittler 


\title{
Large area periodic, systematically changing, multishape nanostructures by laser interference lithography and cell response to these topographies
}

\author{
Erden Ertorer, ${ }^{a, b}$ Fartash Vasefi, ${ }^{c, d}$ Joel Keshwah, ${ }^{e}$ Mohamadreza Najiminaini, ${ }^{c, d}$ Christopher Halfpap, ${ }^{f}$ Uwe Langbein, \\ Jeffrey J. L. Carson, ${ }^{c, d}$ Douglas W. Hamilton, ${ }^{e}$ and Silvia Mittler ${ }^{b}$ \\ a University of Western Ontario (Western University), Biomedical Engineering Program, 1151 Richmond Street, London, Ontario N6A 3K7, Canada \\ bUniversity of Western Ontario (Western University), Department of Physics and Astronomy, 1151 Richmond Street, London, Ontario N6A 3K7, \\ Canada \\ 'Lawson Health Research Institute, Imaging Program, 750 Base Line Road, London, Ontario N6C 2R5, Canada \\ 'University of Western Ontario (Western University), Department of Medical Biophysics, Schulich School of Medicine and Dentistry, \\ 1151 Richmond Street, London, Ontario N6A 3K7, Canada \\ eUniversity of Western Ontario (Western University), Division of Oral Biology, Schulich School of Medicine and Dentistry, 1151 Richmond Street, \\ London, Ontario N6A 3K7, Canada \\ 'RheinMain University of Applied Sciences, Department of Physics, Am Brückweg 26, 6428 Rüsselsheim, Germany
}

\begin{abstract}
The fabrication details to form large area systematically changing multishape nanoscale structures on a chip by laser interference lithography (LIL) are described. The feasibility of fabricating different geometries including dots, ellipses, holes, and elliptical holes in both $x$ - and $y$-directions on a single substrate is shown by implementing a Lloyd's interferometer. The fabricated structures at different substrate positions with respect to exposure time, exposure angle and associated light intensity profile are analyzed. Experimental details related to the fabrication of symmetric and biaxial periodic nanostructures on photoresist, silicon surfaces, and ion milled glass substrates are presented. Primary rat calvarial osteoblasts were grown on ion-milled glass substrates with nanotopography with a periodicity of $1200 \mathrm{~nm}$. Fluorescent microscopy revealed that cells formed adhesions sites coincident with the nanotopography after $24 \mathrm{~h}$ of growth on the substrates. The results suggest that laser LIL is an easy and inexpensive method to fabricate systematically changing nanostructures for cell adhesion studies. The effect of the different periodicities and transition structures can be studied on a single substrate to reduce the number of samples significantly. @ 2013 Society of Photo-Optical Instrumentation Engineers (SPIE) [DOI: 10.1117/1.JBO.18.3.035002]

Keywords: periodic nanostructures; laser interference lithography; Lloyd's interferometer; adhesion and spreading of cells; osteoblasts. Paper 12646RR received Sep. 27, 2012; revised manuscript received Feb. 13, 2013; accepted for publication Feb. 14, 2013; published online Mar. 4, 2013.
\end{abstract}

\section{Introduction}

Nanoscale periodic structures have gained research attention in the last decades as a result of improvements to nanofabrication and nanostructure characterization methods. Their applications include optical gratings, ${ }^{1}$ photonic crystals, ${ }^{2}$ biosensors, ${ }^{3-5}$ and fabrication of surface topographies for microbiological studies. ${ }^{6-10}$ There are several ways to create nanoscale pattern, but most of them, e.g., electron beam lithography (EBL) and focused ion beam lithography (FIB), have low fabrication speed and typically require complex instrumentation. Laser interference lithography (LIL) is a simple and relatively inexpensive technique to create periodic structures over large areas. ${ }^{11,12}$ The Lloyd's interferometer provides a flexible setup for laser LIL with the possibility to create nanoscale structures with different periodicities without additional optical alignment modifications..$^{11,12}$

Most researchers using Lloyd's systems have focused on creating uniform patterns over large areas. ${ }^{13,14}$ However, for some studies, especially the those in the early device development stage, where systematic variation of parameters is necessary, fabrication of a variety of multiple and/or gradually changing structures on a single substrate is the desired goal. A typical

Address all correspondence to: Silvia Mittler, University of Western Ontario (Western University), Department of Physics and Astronomy, London, Ontario, Canada. Tel: 1519661 2111; Fax: 1519661 2033; E-mail: smittler@uwo.ca example is the study of the influence of surface topographies in a systematic fashion in the field of cell biology, where the objective is to examine how cells respond to lines, broken lines and dots or combinations of them on microscopic to nanoscopic scales. ${ }^{15-18}$ Another example is the expanding field of plasmonics, where structures of different sizes and periodicities correspond to different resonance conditions. ${ }^{19-23}$ Furthermore, structures with biaxial periodicities enable cases where it is critical to implement different periodicities on the $x$ - and $y$-axes therefore enabling two measurements on one sample by simply considering the polarization direction of the probing light. ${ }^{24}$ Exploiting biaxial periodicity by polarized light is especially applicable to surface enhanced Raman spectroscopy (SERS) whereby an active substrate with multiwavelength or tunable excitation can be created. ${ }^{24}$ Therefore, fabrication of structures with biaxial periodicity facilitates parallel experimentation, which reduces sample preparation time, decreases the number of independent experiments needed and enables rapid optimization of experimental conditions.

In this paper, we describe an LIL method to create large scale, periodic and biaxial periodic, systematically varying multishape structures on a single substrate. First, we computed the optimal parameters for a Lloyd's mirror interferometer

0091-3286/2013/\$25.00 (C) 2013 SPIE 


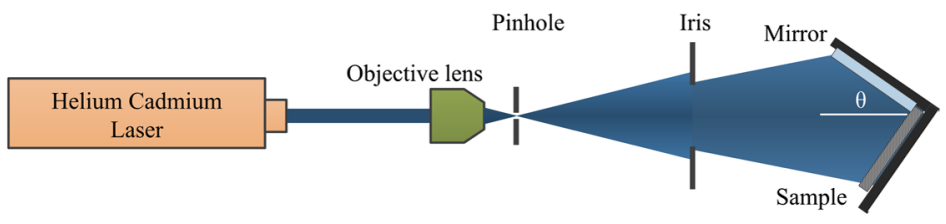

(a)

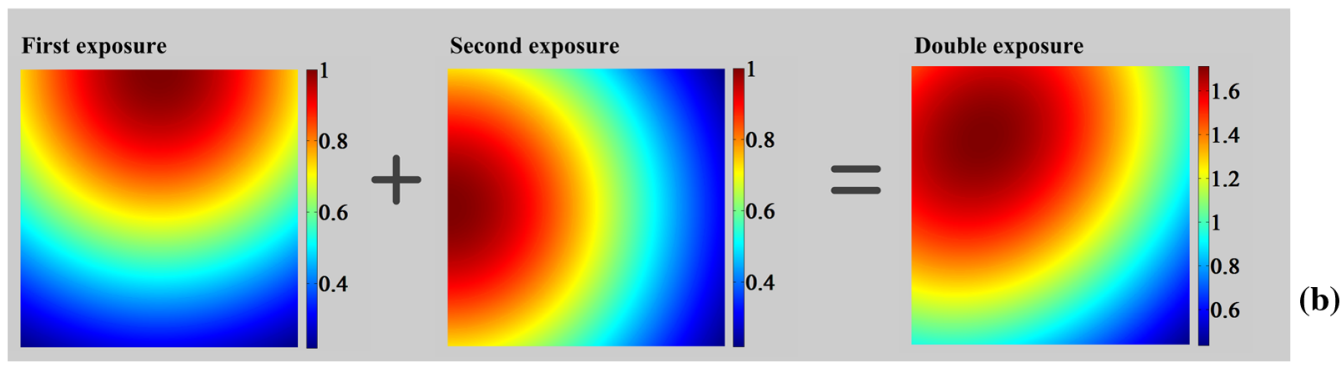

Fig. 1 (a) Lloyd's interferometer setup. (b) Intensity distribution of two individual exposures and the sum due to the double exposure.

implementing a Gaussian laser beam intensity distribution to achieve a large intensity variation along the substrate. Second, we fabricated various multishape periodic and biaxial periodic structures by using different exposure times and exposure angles. The fabricated structures were analyzed with scanning electron microscopy (SEM). Finally, ion-milled glass substrates carrying patterns as surface topography were used for cell adhesion experiments.

\section{Methods}

\subsection{Theory}

Two coherent beams on a plane create an interference pattern. This pattern can be used to expose a photoresist layer to create permanent structures. ${ }^{12}$ The Lloyd's mirror interferometer consists of a mirror placed perpendicular to the sample plane [see Fig. 1(a)]. When a diverging laser beam from a spatial filter (lens and pinhole) illuminates a Lloyd's interferometer, the sample is exposed both to the direct beam and to the reflected beam. The addition of two laser beams creates a light intensity interference pattern on the substrate. The periodicity of the pattern $(\Lambda)$ is given by Lloyd's interferometer equation: ${ }^{12}$

$$
\Lambda=\frac{\lambda}{2 \sin \theta}
$$

where $\lambda$ is the wavelength of the laser beam, and $\theta$ is the angle between the mirror and the axis of the direct laser beam (optical axis of the system).

A Gaussian beam intensity distribution on the substrate for Lloyd's interferometer created by the direct and reflected beams can be represented by Eq. (2): ${ }^{25}$

$$
I=2 I_{0} \exp \left(\frac{-2 x^{2}}{\omega_{r}^{2} \cos ^{2} \theta}+\frac{-2 y^{2}}{\omega_{r}^{2}}\right),
$$

where $I_{0}$ is the peak intensity of the beam, $x$ and $y$ are the distances from the center of the beam, $\omega_{r}$ is the Gaussian beam radius at the distance of the substrate, which is defined as the distance where the intensity decreases to $1 / e^{2}$ from its maximum value and $\theta$ is the angle between the mirror and the optical axes of the experiment (central line of the direct laser beam).
For larger values of magnification and larger distances between the pinhole and sample, the intensity distribution becomes relatively flat along the surface of the mirror geometry yielding uniform structures. This large distance geometry is used by most researchers implementing the Lloyd's mirror interferometer. On the other hand, confining the Gaussian beam or bringing the Lloyd's mirror setup closer leads to a higher variation of the intensity along the sample and mirror. A distinct intensity variation can create various photoresist structures on the substrate. Creating two dimensional structures can be achieved by rotating the photoresist coated substrate by $90 \mathrm{deg}$ and then applying a second exposure. For instance, the intensity distributions for the first, the second, and the total exposure of an experiment with $d=23.5 \mathrm{~cm}, \omega_{0}=$ $1.9 \mathrm{~mm}$, and $\theta=14^{\circ}$ on a sample with a size of $4 \times 4 \mathrm{~cm}^{2}$ are illustrated in Fig. 1(b).

\subsection{Experimental}

A 442-nm wavelength HeCd Laser (Omnichrome Series 74, Model 4074-P-A03, CVI Melles Griot, New Mexico) with a coherence length $L_{c}$ of $30 \mathrm{~cm}$ was operated at $80 \mathrm{~mW}$. The 1.5-mm diameter laser beam passed through a lens-pinhole system built with a $20 \times$ objective lens (Zeiss LD Plan-Neofluar 20X/0.4 Corr $\mathrm{Ph} 2$ ), and a 5- $\mu \mathrm{m}$ pinhole mounted on a three axis stage (Newport Three-Axis Spatial Filter, Model M-900, Newport, California). A time-controlled shutter was situated between the laser and the objective lens to control the exposure time during each experiment. The pinhole was used to remove undesirable components of the laser beam such as donut mode contributions. An iris was used to prevent reflections of the expanded laser beam. The Lloyd's mirror/sample was placed on a two axis stage $(X$ and $\theta$ ) in such a way that the common corner of the sample and the mirror was located on the optical axis. The distance between the sample and the pinhole along the direction of the optical axis was $23.5 \mathrm{~cm}$.

\subsection{Fabrication of Nanostructures}

Experiments were performed with $(2.5 \times 2.5) \mathrm{cm}^{2}$ Fisher Brand microscope slides (soda lime glass) or $(2.5 \times 2.5) \mathrm{cm}^{2}$ fused silica slides (Valley Design Corp., USA) immersed in NanoStrip (Cyantec Inc., California) at $60^{\circ} \mathrm{C}$ for $20 \mathrm{~min}$ to remove 
possible organic and inorganic contamination. In order to increase the adhesion of the photoresist, substrates were silanized with hexamethyldisilazane (HMDS) in an oven (YES-3TA HDMS Oven, Yield Engineering, California). Then substrates were spin-coated at $4000 \mathrm{rpm}$ for $45 \mathrm{~s}$ with 1:4 Shipley S1805 photoresist (Shipley, Massachusetts) diluted with Microposit Thinner Type $\mathrm{P}$ (Shipley, Massachusetts). Samples were soft-baked for $5 \mathrm{~min}$ at $115^{\circ} \mathrm{C}$ on a hot plate. The resulting photoresist thickness was $320 \pm 10 \mathrm{~nm}$ found by analysis of electron microscopy images of FIB cut samples. To achieve the photoresist structures which carry the same periodicity on both axes, samples were fabricated with a mirror-laser beam axis angle $\theta \sim 15 \mathrm{deg}$, yielding a periodicity $\Lambda$ of $\sim 800 \mathrm{~nm}$. The sample was exposed first for $18 \mathrm{~s}$, rotated by $90 \mathrm{deg}$ and exposed a second time for $18 \mathrm{~s}$. A second sample was exposed first for $18 \mathrm{~s}$, then $12 \mathrm{~s}$ after the $90 \mathrm{deg}$ rotation.

For the biaxial periodic structures, with different periodicities in $x$ - and $y$-direction, $\theta$ was set to $\sim 11 \mathrm{deg}$, yielding a periodicity $\Lambda$ of $\sim 1200 \mathrm{~nm}$. After the first exposure, (for various times) the sample was developed, then placed in the Lloyd's setup rotated by $90 \mathrm{deg}$ with respect to the first exposure and exposed a second time (for various times) at an angle $\theta$ of $\sim 15$ deg to obtain a periodicity $\Lambda$ of $\sim 800 \mathrm{~nm}$.

Substrates were developed in MF319 developer (Shipley, Massachusetts). Symmetric structures were developed once after the two exposures. Biaxial structures were developed twice, once after the first exposure and then again after the second exposure. The first development was $45 \mathrm{~s}$; the second development was $20 \mathrm{~s}$. After each development, substrates were rinsed with copious amounts of deionized water and dried under nitrogen. Finally, samples were hard-baked for $10 \mathrm{~min}$ at $115^{\circ} \mathrm{C}$ on a hot plate. All the procedures were performed in a clean room facility at $21.5 \pm 0.5^{\circ} \mathrm{C}$, relative humidity of $30 \pm 10 \%$, and under yellow light.

The photoresist patterns were also used as etching masks for the fabrication of metallic or silicon nanofeatures. A 300-nm photoresist layer was spun on a p-type silicon substrate (500 $\mu \mathrm{m}$ thickness) with a 30-nm chromium layer deposited by an electron-beam evaporation system. The photoresist acted as an etching mask for the chromium layer resulting in a chromium pattern identical to the photoresist pattern. The patterned chromium nanostructures acted as a masking layer for silicon ion etching. Therefore, the pattern and objects fabricated lithographically in photoresist were transferred to the chromium layer by wet etching as well as the silicon wafer by deep reaction ion etching (DRIE; Alcatel 601E Deep Silicon Etch, France).

Fused silica samples carrying line and dot structures at a periodicity of $1200 \mathrm{~nm}$ in photoresist were ion milled (Vacu Tec Plasma Systems: control unit CPU 500, matching Plasmamatch; ENI: RF-generator ACG-3XL; Witney, Oxfordshire, United Kingdom) with SF6 at a gas flow rate of $20 \mathrm{sccm}, 200 \mathrm{~W}$ plasma power and a pressure of $\sim 8 \mathrm{~Pa}$. Under these conditions, a surface topography with a profile depth of $\sim 100 \mathrm{~nm}$ was achieved. The structures were analyzed with SEM after ion milling to acquire the profile depth. The fused silica nanotopographic samples were used in the cell studies.

\subsection{Osteoblast Culture}

All studies involving rats were performed in compliance with the University Council on Animal Care at the University of Western Ontario under approved protocols. Rat calvarial osteoblasts (RCOs) were obtained from newborn rat calvariae and cultured as previously described. ${ }^{26}$

\subsection{Immunocytochemistry}

Osteoblasts were plated at a density of 39 cells $/ \mathrm{mm}^{2}$ and were fixed in $4 \%$ paraformaldehyde at $24 \mathrm{~h}$ post-seeding. Samples were then stained with rhodamine-conjugated phalloidin (Sigma-Aldrich, Canada), 4,6-diamidino-2-phenylindole dihydrochloride (DAPI) (Sigma-Aldrich, Canada), and vinculin (Sigma-Aldrich, Canada) as previously described. ${ }^{7,26}$ Images were captured from each surface on an AxioScope microscope (Zeiss, Germany) using an Axiocam digital camera and AxioImager software.

\section{Results}

\subsection{2-D Multishaped Structures}

To correlate nanostructure shape with corresponding exposure and light intensity conditions, nine regions on each sample were chosen and characterized. Figure 2 shows the exposure intensity map as well as the selected regions where SEM images were taken. The first region $R_{(0,0)}$ was chosen at the origin of the $x y$-coordinate system and corresponded to the location with the highest exposure intensity. The remaining eight regions were distributed 10 and $15 \mathrm{~mm}$, respectively away from the origin in both $x$ - and $y$-directions. For instance, $R_{(10,15)}$ represented the region that was displaced $10 \mathrm{~mm}$ in the $x$-direction and $15 \mathrm{~mm}$ in the $y$-direction relative to the origin. As Fig. 2 shows, the nanostructure shape correlated directly with the exposure time and the beam intensity at each region.

In the case of the equivalent double exposure of sample 1, it was expected from Eq. (1) that the exposure intensity dropped by $6 \%$ and $14 \%$ at a distance of 10 and $15 \mathrm{~mm}$ away from the highest value at the origin, respectively. The diagonal in the $x y$-coordinate system was an axis of symmetry for the substrate. At the origin, $\left[R_{(0,0)}\right.$ in Fig. 2(b)], round pillars were observed. Moving from the origin to $R_{(10,10)}$ the dot diameter gradually increased and some were connected. After passing $R_{(10,10)}$, connected dots formed holes and at $R_{(15,15)}$ the exposure was not high enough for the developer to etch the photoresist through to the substrate.

For areas off the axis of symmetry, either the $x$ - or the $y$ - component, the structures were elongated with respect to the other direction: elongated meaning that when lines form, the lines were along the $x$-direction on the right hand side of the symmetry line and along the $y$-direction on the left hand side of the symmetry line. The directions of the long axes of ellipses were analogous. The result was that $R_{(10,0)}$ contained objects elongated in the $x$-direction and $R_{(0,10)}$ contained structures elongated in the $y$-direction.

The elongation trend continued into wavy lines in either of the directions. The off-diagonal areas $R_{(10,15)}$ and $R_{(15,10)}$ contained broad wavy lines that were well-separated from one another. The ensemble of the wavy lines can be interpreted as a prestructure for the hole array located on the axis of symmetry at $R_{(15,15)}$ obtained with an exposure of lower intensity.

Another observation from Fig. 2 was the fact that in the high intensity areas, separated islands or nanodots were formed and with decreasing illumination intensity, the structures became 
Sample 1

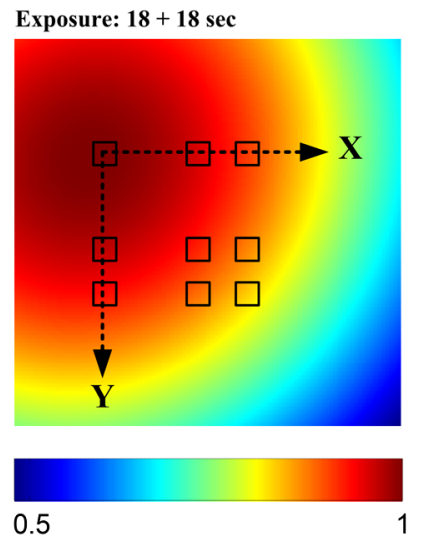

(a)

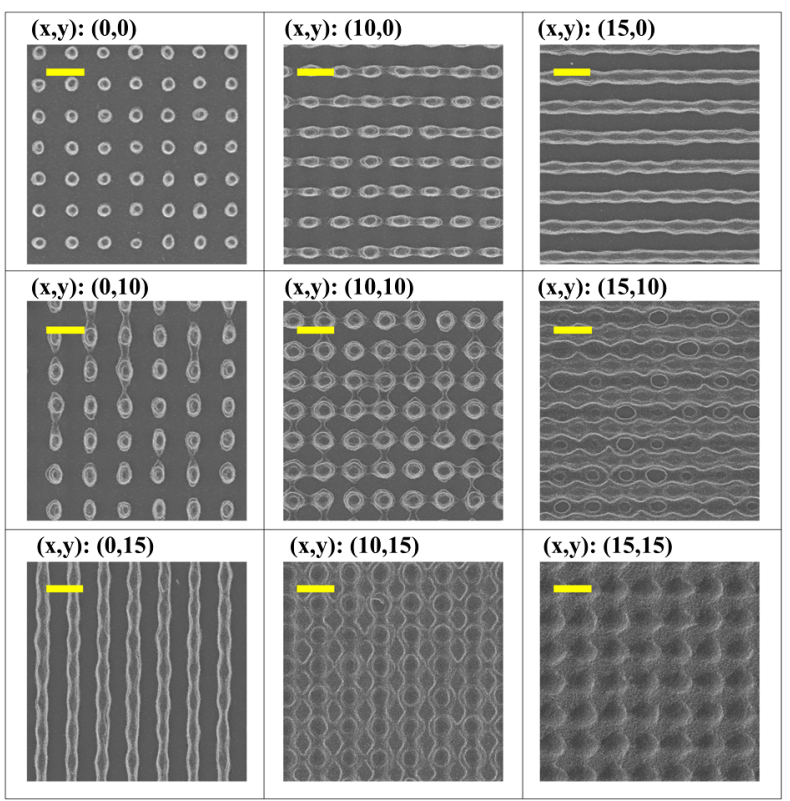

(b)

Sample 2

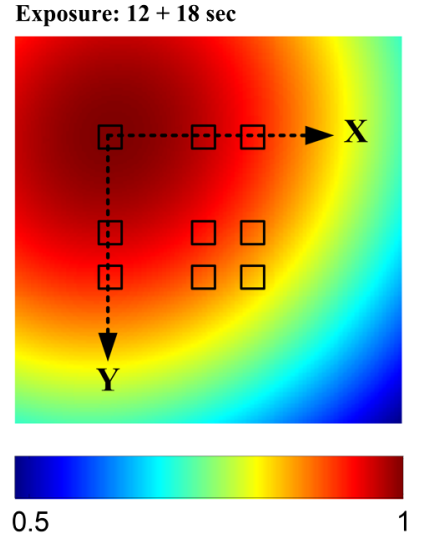

(c)

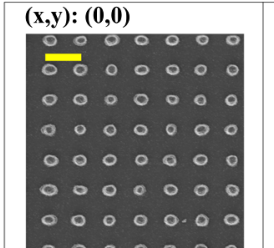

$(\mathbf{x}, \mathbf{y}):(\mathbf{0 , 1 0})$

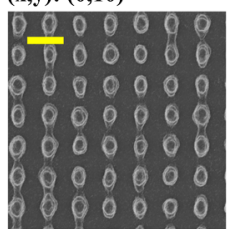

$(\mathbf{x}, \mathbf{y}):(0,15)$

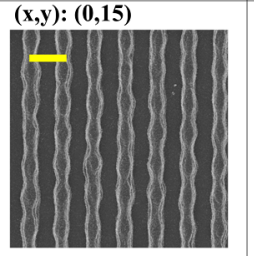

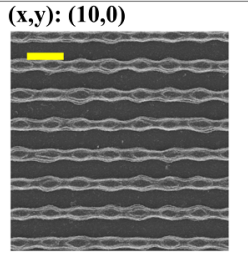

$(x, y):(10,10)$

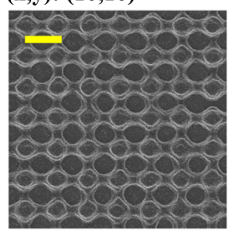

$(\mathrm{x}, \mathrm{y}):(10,15)$

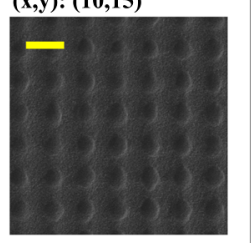

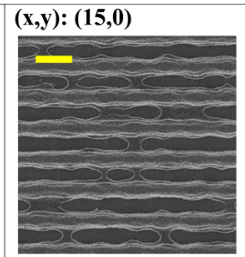

$(x, y):(15,10)$

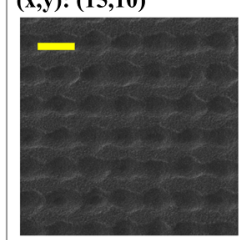

$(\mathbf{x}, \mathbf{y}):(15,15)$

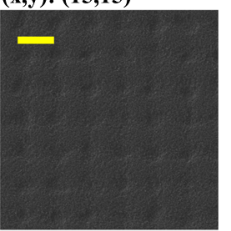

(d)

Fig. 2 Illumination intensity maps and SEM images of photoresist patterns of samples fabricated with LIL. (a) Cumulative illumination intensity map for $18+18 \mathrm{~s}$ of exposure. (b) SEM images of nine selected regions from samples receiving illumination corresponding to (a). Location of each image corresponds to the location in the accompanying illumination intensity map. Scale bars represent $1 \mu \mathrm{m}$. (c) Cumulative illumination intensity map for $18+12$ s exposure. (d) SEM images of nine selected regions from samples receiving illumination corresponding to (b).

connected with sharp linear features. Further decreases in the intensity led to a shape inversion, i.e., the formation of nanoholes.

The use of different exposure times when the substrate was in the $x$ - and $y$-orientations in sample 2 resulted in samples with unique nanostructures within each region. Figure 2(d) clearly shows that the overall structure was not symmetric with respect to the diagonal. For example, the nanostructures in $R_{(10,0)}$ had a linear shape, while the nanostructures in $R_{(0,10)}$ were nominally oval-shaped. Furthermore, the nanostructures formed at the $R_{(0,0)}$ in Fig. 2(d) were slightly elongated in the $x$-direction, making them elliptical, while the nanostructures observed at $R_{(0,0)}$ in Fig. 2(b) were nearly circular.

\subsection{Nanostructures with Biaxial Periodicity}

The combination of different exposure time, different exposure angles for the $x$ - and $y$-axes, and post processing resulted in a wide variety of structures with controllable biaxial periodicity and morphology, including dots, holes, ellipses, elliptical holes, lines, and wavy lines. For instance, Fig. 3(a) shows elliptical nanodots in photoresist on a glass substrate with $1200 \mathrm{~nm}$ periodicity on the $x$-axis and 800 -nm periodicity on the $y$-axis that resulted from exposure sets of $20+20 \mathrm{~s}$. Figure 3(b) shows elliptical nanoholes in photoresist on a glass substrate with similar biaxial periodicity to the structure in Fig. 3(a) that resulted from an exposure set of $22+18 \mathrm{~s}$. Figure 3(c) and 3(d) shows 


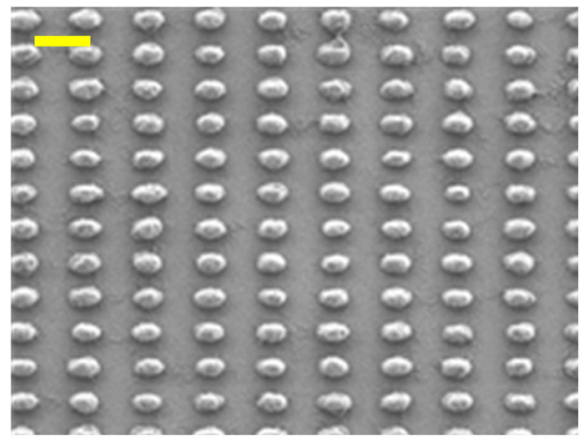

(a)

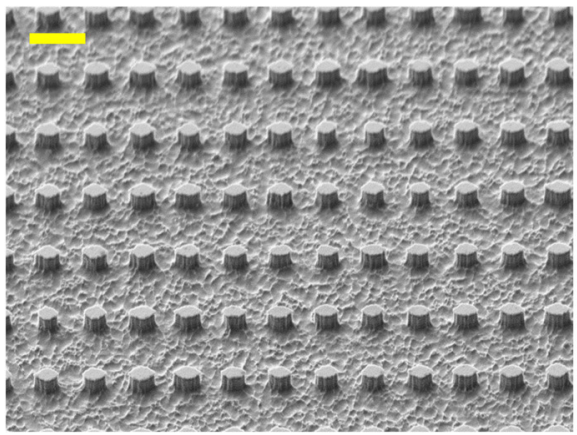

(c)

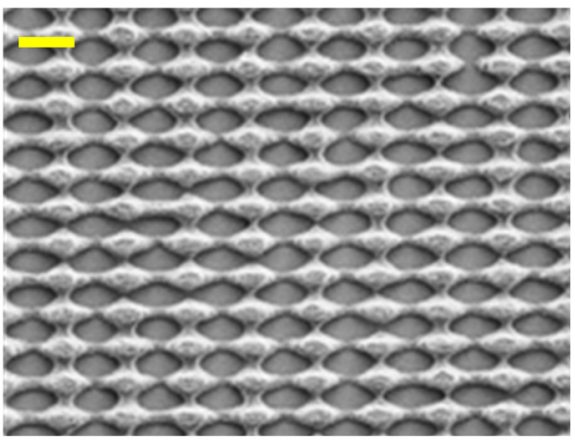

(b)

Che000000000000000000

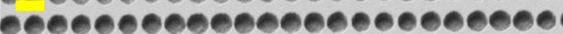

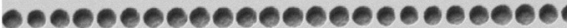
- 0000000000000000001

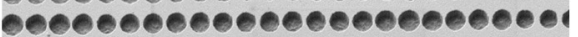
- 000000000000000000 r - 000000000000000000004

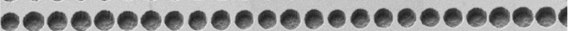
- 0000000000000000001 -

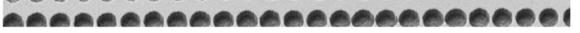

(d)

Fig. 3 SEM images of nanostructures with biaxial periodicity (a) ellipses (in photoresist), (b) elliptical holes (in photoresist), (c) round pillars (in silicon), and (d) round holes (in silicon). Scale bars represent $1 \mu \mathrm{m}$.

silicon nanopillar and nanohole structures with a height equal to $300 \mathrm{~nm}$. In order to achieve $800 \mathrm{~nm}$ spacing on the $x$-axis and $1200 \mathrm{~nm}$ on the $y$-axis, the exposures sets of $15+25 \mathrm{~s}$ and $11+$ $22 \mathrm{~s}$ were used to form nanodots and nanoholes, respectively.

\subsection{Cell Response to Nanotopographies}

We tested the influence of the nanotopographies, explicitly on a dot pattern [Fig. 4(a)] and on a line pattern [Fig. 4(b)]. Cell adhesion and spreading of primary rat calvarial osteoblasts (Fig. 5) was tested. The vinculin stain (green) depicts the adhesions of the cell onto its substratum, whereas the f-actin (red) depicts actin microfilaments and hence relays information on cell morphology. The cell nuclei were stained with DAPI (blue). In the control experiments on smooth surfaces (no pattern) outside the structure of the substrates (Fig. 5, top row), adhesion sites

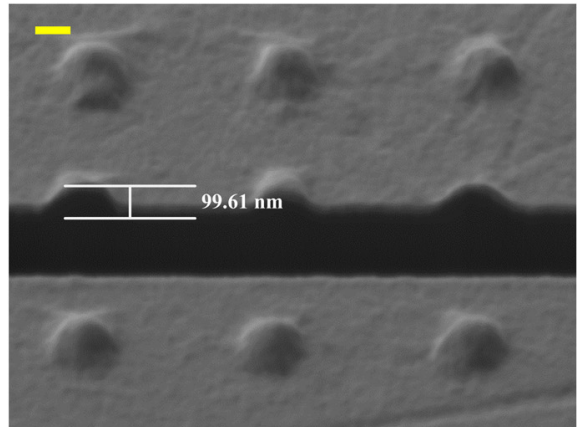

(a) (vinculin) were randomly oriented, as was cell spreading (f-actin). On the dot patterns (Fig. 5, middle row), adhesion sites were formed on the peaks of the topographies, which was evident from the dot patterning apparent in the vinculinstained image (see inset). The cell morphology did not conform to the shape of the topography. On the $1200 \mathrm{~nm}$ spaced lines (Fig. 5, bottom row), osteoblasts formed densely adhesions in parallel with the long axis of the topography (vinculin), and the overall morphology (f-actin) was also aligned with the groove direction.

\section{Discussion}

Laser LIL is a straight-forward, flexible, and inexpensive method to create a variety of nanostructures. Utilizing Eq. (2), experimental parameters can be selected to achieve a

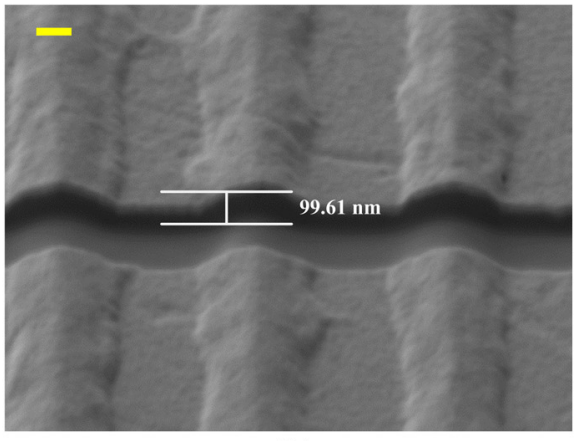

(b)

Fig. 4 SEM images of a dot (a) and a line (b) pattern on ion-milled glass substrates coated with 30-nm thick gold for SEM imaging. These example samples were cut with a focused ion beam to estimate the depth. The tilt corrected cursor height is $\sim 100 \mathrm{~nm}$ excluding the gold coating. Scale bars represent $100 \mathrm{~nm}$. 

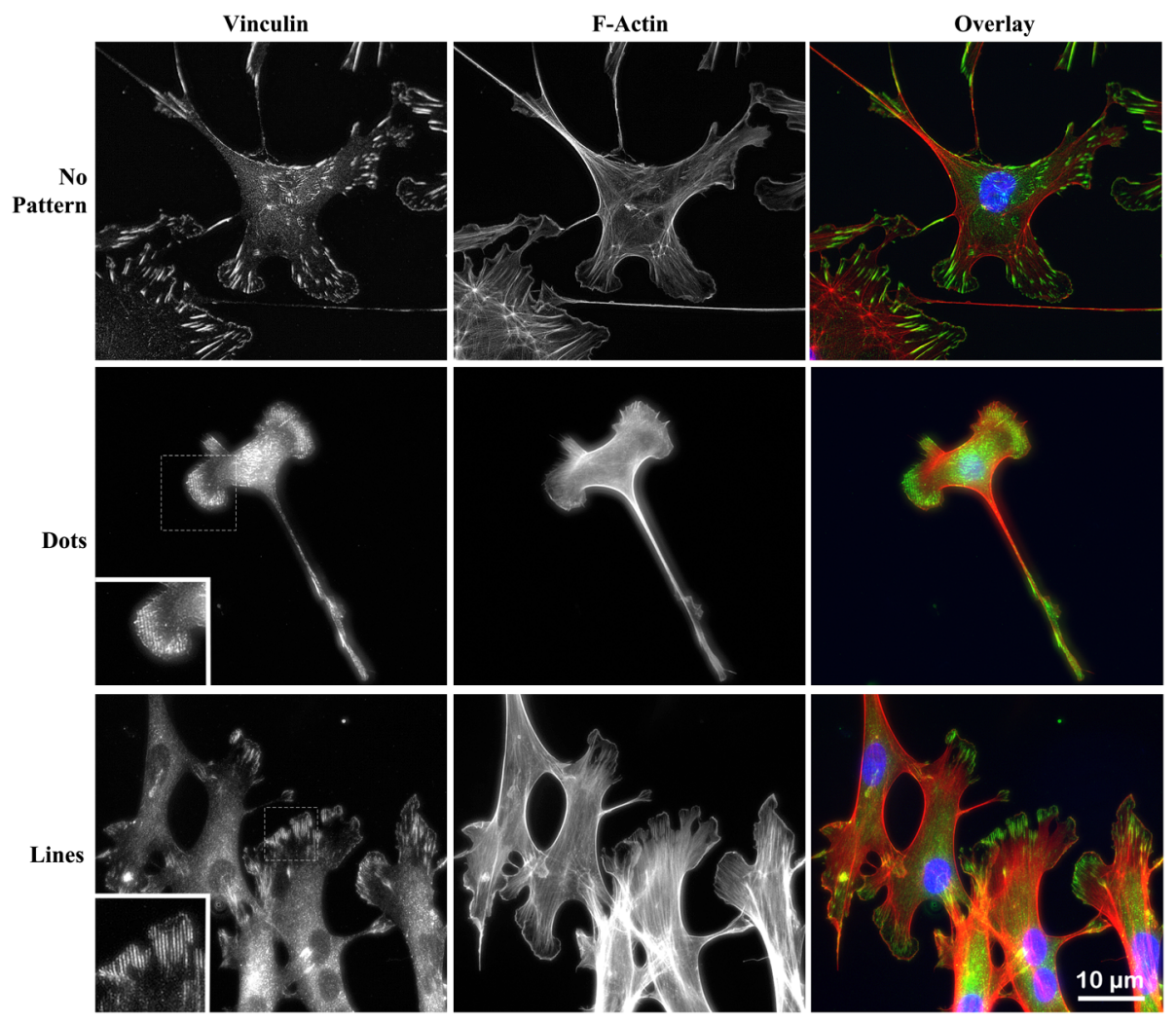

Fig. 5 Rat calvarial osteoblasts cultured on smooth surfaces (top row), $1200 \mathrm{~nm}$ spaced nanodots (middle row) and $1200 \mathrm{~nm}$ spaced nanolines (bottom row). Adhesion sites were stained with vinculin (left column). Actin microfilaments were stained with f-actin (middle column). Overlay of the vinculin, $\mathrm{f}$-actin and DAPI (nuclei) are depicted as green, red, and blue, respectively (right column). Insets show high resolution images of marked areas.

cumulative illumination pattern on the photoresist that leads to a wide range of patterns. We used a short distance between the pinhole and the substrate to create a high intensity gradient along the surface, which systematically yielded a variety of structures with morphologies, such as dots, holes, ellipses, lines, and transitions between morphologies. Changing the stage angle and the exposure time for two different exposure axes created structures with biaxial periodicities.

EBL and FIB technologies provide the freedom to fabricate more arbitrary structures with finer features, however, they both require expensive setups and high operating costs and work in series. Therefore, creating large area structures, such as $15 \times 15 \mathrm{~mm}^{2}$, as shown in this study, EBL and FIB will be impractical in terms of the time requirements for the fabrication. Therefore, LIL is a fast, inexpensive and "parallel operating" alternative to create systematically changing structures as well as periodic and biaxial structures.

Osteoblasts showed a differential response to $1200 \mathrm{~nm}$ spaced dots and lines, with qualitative changes in the arrangement and distribution of adhesions sites. In addition the cell morphology is strongly influenced by the form and orientation of the nanotopography. The relative importance on topographic regulation of focal adhesion size and stability in osteoblasts has received much attention. Biggs et al. previously demonstrated that nanopits reduced the total cell area occupied by adhesion sites in osteoblasts ${ }^{27}$ and that square and hexagonal nanopit arrays resulted in formation of focal complexes. ${ }^{28}$ Our results show that nanotopographies strongly influence the patterns of adhesion formation in osteoblasts.

All these structures can also be fabricated in thick and thin metal films placed on a planar substrate to obtain plasmonic structures. ${ }^{13,29,30}$ The localized surface plasmon resonances (LSPR) of these metallic arrays are a function of material, size, shape, spacing and periodicity of the structures. The size and the spacing of the structures could be adjusted by fine tuning the exposure times and the exposure angle. Combined bimetallic nanostructures, consisting of two different metal layers, can provide additional tunability to the LSPR properties. ${ }^{29}$ Nanohole and nanodot arrays with biaxial periodicity provide intrinsically two different LSPR frequencies due to the possible choice of the probing polarization direction of the light. Meanwhile, there is a multitude of LSPR sensors and devices proposed, simulated and experimentally demonstrated, that implement above described parameters. A single chip carrying systematically changing nanostructures can help to quickly find the optimal nanostructure for the problem at hand to be solved with plasmonics. If an optical experiment is performed on elongated structures, the polarization of the probing light is exploited to detect the morphometric differences. The sharp linear features could act as an active substrate with hot spots in the case of a gold or silver nanostructure applied in SERS or fluorescence enhanced spectroscopy.

Due to the geometrical limitations, the Lloyd's laser interferometer has a limited surface coverage and for a wavelength of $442 \mathrm{~nm}$ the fabricated structures were limited to roughly 600 to $1200 \mathrm{~nm}$ periodicity. One potential improvement here would be a sample mount allowing the adjustment of the angle between the mirror and the sample. This will add an additional degree of freedom and would extend the usable range of coverage and periodicity. ${ }^{14}$ However, ultra-fine structures may require antireflective coatings on the sample to enhance optical performance. ${ }^{12}$ In order to reach smaller periodicities, a shorter 
wavelength laser can be utilized. For example, extreme ultraviolet LIL (EUV-IL) has produced feature sizes down to $10 \mathrm{~nm}^{31}$

Some nonuniformity within structures was observed, which was due to photoresist connections between adjacent features, especially for sample 1 at locations $R_{(0,10)}, R_{(10,10)}$, and $R_{(10,0)}$. These samples could be treated with oxygen plasma after sample development, which would result in a removal of the connections between adjacent nanostructures and individual features with a higher degree of uniformity.

Although structures created with LIL are limited to grooves, holes and dots, nanolithography employing micro-lens arrays has been shown capable of fabricating periodic arrays of arbitrary structures. ${ }^{32,33}$ The technique utilizes an array of focal points from a laser beam that also enables parallel patterning. ${ }^{33,34}$ However, it requires micro-lens array fabrication prior to the fabrication of the intended nanostructures. The periodicity of nanostructure array depends greatly on spacing between adjacent micro-lenses. ${ }^{33,34}$ It had been shown that plasmonic nanolithography can reach down to $12 \mathrm{~nm}$ feature size. However, that techniques is limited to periodic structures. ${ }^{35}$

Surface topography features that systematically change in pattern have become an important tool to probe the limits of cell sensing, as well as to increase our understanding of how cells become activated. ${ }^{36}$ Adhesion sites on cells are important for activation of downstream signaling cascades that are responsible for regulation of growth, survival, migration, and differentiation. ${ }^{7,36}$ By selecting the most appropriate topographies, it is conceivable that implants and tissue-engineered devices could be significantly improved. Moreover, from a basic science standpoint, devices fabricated with LIL may be particularly good to assess the response of primary cell cultures, where heterogeneity in growth and response to external stimuli, which is a common place in human cells isolated from different patients, can complicate interpretation of results. Therefore, to be able to have one substrate with a variety of nanostructure pattern and their transitions allow comparison of cell responses to multiple cues at the same time.

We assume that the periodicity and the depth of structures (the aspect ratio) could provide a different behavior for cell adhesion to the substrate; therefore, in future work, the effect of the various structure depths and periodicities will be studied. The behavior of various cell types (e.g., stem cells) as well as the behavior on transitional nanostructures (between two structures, such as broken lines which is between lines and dots) should be investigated. Aside from topography, the surface chemistry can also be controlled by various surface functionalization techniques, which opens up the possibility to study the combinatorial effect of topography and surface chemistry on cell adhesion and morphology. These directions of study should provide a better understanding of implant surfaces with respect to growth and nongrowth surfaces for particular cell types.

\section{Conclusion}

We have shown that laser LIL is a powerful method to fabricate large area, periodic, biaxial periodic and systematically changing structures. In comparison to FIB and EBL, LIL is straightforward, quick and inexpensive. The systematically changing structures allow optimization processes, e.g., for plasmonic structures with different resonance conditions. The system can be further developed for smaller feature sizes by employing a laser source with a smaller wavelength and for covering even larger areas.

Cell culturing studies show that primary rat calvarial osteoblasts respond to surface topographies and allow us to learn controlling their behavior on surfaces. Having biaxial periodic and systematically changing structures on the same substrate allows studying various structures with a single substrate to decrease the amount of samples and time in finding an optimum topography for a certain cell response.

\section{Acknowledgments}

The authors thank the Western Nanofabrication Facility for hosting the Lloyd's mirror interferometer and for the help with SEM imaging and FIB. We thank Abdollah Hassanzadeh and Bozena Kaminska for helpful discussions. The NSERC BiopSys Strategic Network is recognized for financial contributions. NSERC and CFI are acknowledged for their financial support in form of Discovery Grants and equipment funding, respectively. E.E. thanks the OGS (Ontario Graduate Scholarship) Program for financial aid. S.M. thanks the Canadian Government for support through the CRC Program.

\section{References}

1. A. Akou et al., "Soft lithographic patterning of spin crossover complexes. Part 2: stimuli-responsive diffraction grating properties," J. Mater. Chem. 22(9), 3752 (2012).

2. A. Reinhard et al., "Strongly correlated photons on a chip," Nat. Photon. 6(2), 93-96 (2012).

3. C. Escobedo et al., "Flow-through vs flow-over: analysis of transport and binding in nanohole array plasmonic biosensors," Anal. Chem. 82(24), 10015-10020 (2010).

4. S. M. H. Rafsanjani et al., "Theoretical proposal for a biosensing approach based on a linear array of immobilized gold nanoparticles," J. Appl. Phys. 107(9) 094303 (2010).

5. H. Jiang et al., "A biosensor based on periodic arrays of gold nanodisks under normal transmission," Sensors Actuat. A: Phys. 189, 474-480 (2013).

6. D. W. Hamilton and D. M. Brunette, “'Gap guidance' of fibroblasts and epithelial cells by discontinuous edged surfaces," Exp. Cell Res. 309(2), 429-437 (2005).

7. D. W. Hamilton and D. M. Brunette, "The effect of substratum topography on osteoblast adhesion mediated signal transduction and phosphorylation," Biomaterials 28(10), 1806-19 (Apr. 2007).

8. F. Kantawong et al., "Proteomic analysis of human osteoprogenitor response to disordered nanotopography," J. Royal Soc. Interface Royal Soc. 6(40), 1075-1086 (2009).

9. E. Kokubu et al., "Modulation of human gingival fibroblast adhesion, morphology, tyrosine phosphorylation, and ERK 1/2 localization on polished, grooved and SLA substratum topographies," J. Biomed. Mater. Res. Part A 91(3) 663-670 (2009).

10. E. Lamers, J. Te Riet, and M. Domanski, "Dynamic cell adhesion and migration on nanoscale grooved substrates," Eur. Cell. Mater. 23, 182194 (2012).

11. Q. Xie et al., "Fabrication of nanostructures with laser interference lithography," J. Alloys Compds. 449(1-2), 261-264 (2008).

12. H. Wolferen and L. Abelmann, "Laser interference lithography," in Science, pp. 133-148, Nova Publishers, Hauppauge, NY (2011).

13. J. W. Menezes et al., "Large-area fabrication of periodic arrays of nanoholes in metal films and their application in biosensing and plasmonicenhanced photovoltaics," Adv. Funct. Mater. 20(22), 3918-3924 (2010).

14. I. Wathuthanthri, W. Mao, and C.-H. Choi, "Two degrees-of-freedom Lloyd-mirror interferometer for superior pattern coverage area.," Opt. Lett. 36(9), 1593-1595 (2011).

15. T. P. Kunzler et al., "Systematic study of osteoblast response to nanotopography by means of nanoparticle-density gradients," Biomaterials 28(33), 5000-5006 (2007). 
16. Y. L. Khung, G. Barritt, and N. H. Voelcker, "Using continuous porous silicon gradients to study the influence of surface topography on the behaviour of neuroblastoma cells," Exp. Cell Res. 314(4), 789-800 (2008).

17. D. Hamilton et al., "Migration of periodontal ligament fibroblasts on nanometric topographical patterns: influence of filopodia and focal adhesions on contact guidance," PLoS One 5(12), e15129 (2010).

18. T. P. Kunzler et al., "Systematic study of osteoblast and fibroblast response to roughness by means of surface-morphology gradients," Biomaterials 28(13), 2175-2182 (2007).

19. K. L. Kelly et al., "The optical properties of metal nanoparticles: the influence of size, shape, and dielectric environment," J. Phys. Chem. B 107(3), 668-677 (2003).

20. Y. Xia, N. J. Halas, and G. Editors, "Shape-controlled surface plasmonic nanostructures," MRS Bull. 30(5), 338-344 (2005).

21. P. K. Jain et al., "Calculated absorption and scattering properties of gold nanoparticles of different size, shape, and composition: applications in biological imaging and biomedicine," J. Phys. Chem. B 110(14), 7238-7248 (2006).

22. P. Rooney et al., "Control of surface plasmon resonances in dielectrically coated proximate gold nanoparticles immobilized on a substrate," Phys. Rev. B 77(23), 1-9 (2008).

23. Q. Min et al., "Substrate-based platform for boosting the surfaceenhanced Raman of plasmonic nanoparticles," Opt. Express 19(2), 1648-1655 (2011).

24. F. Eftekhari et al., "Polarization-dependent sensing of a self-assembled monolayer using biaxial nanohole arrays," Appl. Phys. Lett. 92(25), 253103 (2008).

25. I. Byun and J. Kim, "Cost-effective laser interference lithography using a $405 \mathrm{~nm}$ AlInGaN semiconductor laser," J. Micromech. Microeng. 20(5), 055024 (2010).
26. R. J. Miron et al., "The effect of enamel matrix proteins on the spreading, proliferation and differentiation of osteoblasts cultured on titanium surfaces," Biomaterials 31(3), 449-460 (Jan. 2010).

27. M. J. P. Biggs et al., "The effects of nanoscale pits on primary human osteoblast adhesion formation and cellular spreading," J. Mater. Sci. Mater. Med. 18(2), 399-404 (2007).

28. M. J. P. Biggs et al., "The use of nanoscale topography to modulate the dynamics of adhesion formation in primary osteoblasts and ERK/ MAPK signalling in STRO-1+ enriched skeletal stem cells," Biomaterials 30(28), 5094-5103 (2009).

29. C. H. Liu et al., "Bimetallic structure fabricated by laser interference lithography for tuning surface plasmon resonance," Opt. Express 16(14), 10701-10709 (2008).

30. D. Xia, "A facile approach to directed assembly of patterns of nanoparticles using interference lithography and spin coating," Nano Lett. 4(7), 1295-1299 (2004).

31. V. Auzelyte et al., "Extreme ultraviolet interference lithography at the Paul Scherrer Institut," J. Micro/Nanolithogr. MEMS MOEMS 8(2), 021204 (2009).

32. J. Kato et al., "Multiple-spot parallel processing for laser micronanofabrication," Appl. Phys. Lett. 86(4), 044102 (2005).

33. Y. Lin et al., "Ultrafast-laser-induced parallel phase-change nanolithography," Appl. Phys. Lett. 89(4), 041108 (2006).

34. C. S. Lim et al., "Microlens array fabrication by laser interference lithography for super-resolution surface nanopatterning," Appl. Phys. Lett. 89(19), 191125 (2006).

35. H. Liu and J. Teng, "Plasmonic nanolithography: towards next generation nanopatterning," J. Molec. Eng. Mater. 1(1), 1250005 (2013).

36. J. Y. Lim and H. J. Donahue, "Cell sensing and response to micro- and nanostructured surfaces produced by chemical and topographic patterning," Tissue Eng. 13(8), 1879-1891 (2007). 